\title{
Segmentation and Detection of Brain Tumor Using 2D and 3D Image Processing
}

\author{
Aathithya $\mathrm{S}^{\mathrm{a}, 1}$, Kavya $\mathrm{S}^{\mathrm{b}}$, Malavika $\mathrm{J}^{\mathrm{b}}$, Aishwarya L R ${ }^{\mathrm{b}}$ And Jeevika $\mathrm{M}^{\mathrm{b}}$ \\ ${ }^{a, b} U G$ scholar.Department of E\&I, \\ Panimalar Engineering College, India
}

\begin{abstract}
Brain tumor is a growth of abnormal cells in the brain that can be either benign or malignant. It tends to affect people irrespective of age group. Thus earlier detection and treatment of brain tumor is needed for quick recovery. Doctors use Magnetic Resonance Imaging (MRI) to identify the intensity of tumor. The MRI images are pre-processed and then Deep learning neural network and spearman algorithms are used to provide segmentation of images. The segmented images are grouped using Fuzzy clustering technique. The CNN and SVM Classifiers are used to classify and detect the size of tumor cell. On comparison, it was found that CNN classifiers are more accurate, sensitive and yields high performance as compared to SVM classifiers for both $2 \mathrm{D}$ and $3 \mathrm{D}$ images.
\end{abstract}

Keywords. Magnetic Resonance Imaging (MRI), Preprocessing, Spearman algorithm, Segmentation, Fuzzy Clustering, Convolution Neural Network (CNN), Support Vector Machine (SVM).

\section{Introduction}

Tumor is detected in a human body due to the presence of cancer cells whose impact will be highly pervasive. Brain tumor is a disease which tends to attack people of any age group. It is an extra growth inside the restricted area of the brain due to which a pressure is created and thus becomes a life threatening phenomena and needs to be screened. The tumor cells can be identified with the help of medical imaging system which involves Segmentation and Classification [1]. Using MRI Growth detection, Analysis and Location can be found. After recognizing the tumor cells, Machine Learning Algorithm will be introduced.

MRI consists of strong magnetic field which will excite the atoms in the brain to move within the field. When a RF current is passed, the atoms are stimulated and they come out of equilibrium producing an opposite pressure to magnetic field. When it is stopped, the atoms realign with the magnetic field and the energy released is also noted. This realignment time and the energy released will depend on the nature of the molecule. Thus, the magnetic property determines the characteristics of the tissue.

\footnotetext{
${ }^{1}$ Aathithya.S, UG scholar.Dept of E\&I Panimalar Engineering College,India Email:aathithya@gmail.com
} 
MRI Sectional anatomy of brain is done using Neuron Imaging, where the 3 planes can be inferred namely: Axial plane, Sagittal plane, Coronal plane. Coronal read will have the information regarding the patient's substance.

\section{Existing System}

2D MRI image of brain is obtained, and pre-processing is done to improve signalnoise ratio, to smooth the inner part of region and to preserve its edges. The preprocessed MRI is converted to binary image [2]. The pixel value of image greater is mapped as black. The black pixel is extracted and is found to be a brain MRI mask. Similar regions of the image are grouped using Edge Detection. After which classifiers are inverently used in order to detect the tumor cells. Classifiers are fed with Data structure and the output thus obtained gives an Exact Detail about the tumor cells.

\section{Proposed System}

Volumetric imaging or 3D MRI image gives a fine anatomic detail [3], and thus the radiologist can manipulate images by emphasizing the areas of interest for a better understanding of the anatomic relationships.

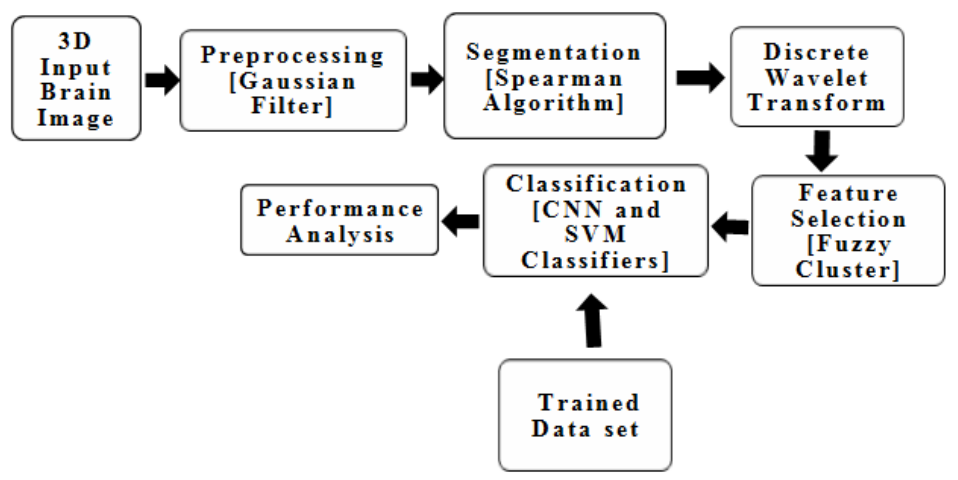

Figure 1. Block diagram of proposed system

The advantage of using 3D imaging is that it has high sensitivity and specificity. Most important is that the exact size and location of tumor can be found.

\subsection{Preprocessing}

In our proposed system, we use the Gaussian filter to preprocess the image by blurring, reducing the contrast and noise of the image [4]. Its impulse response is a Gaussian function which helps us to partially identify the tumor region.

\subsection{Segmentation}

Utilization of gray scale co-occurrence matrix feature is implemented for the extraction of the potential component vectors for image based disease diagnosis [5]. 
Here, in this section the preprocessed MRI Brain image is converted to binary format and the accurate region of the tumor is recognized using the spearman algorithm.

\subsection{Discrete Wavelet Transform}

In contrast to Fourier transforms, the discrete wavelet transform has a temporal resolution which provides us with both frequency and location information. This representation can be utilized to characterize the transient events, minimize noise, compress data, and perform many other operations. Thus the DWT is used for the conversion of continuous signals as discrete samples.

\subsection{Fuzzy C-means algorithm}

Edge detection is an image processing technique for finding the boundaries of the objects within images using Fuzzy C-means algorithm. Fuzzy C-means algorithm is a type of technique for data clustering in which each data point is combined as a part of mean shift algorithm and density estimation. Thus the obtained discrete signal segmented images are enhanced based on the optimization technique implemented with the SVM or CNN classifiers.

\subsection{Feature Extraction}

Feature Selection is the means of choosing a subset of relevant features (variables, predictors) for using in model construction. In specific, feature selection has been successfully implemented in medical applications, where it can not only reduce dimensionality but also help us figure out the root cause of a disease. Feature extraction is the process of altering the existing data to get more precise features. Part Extraction is useful to understand where the tumor is located. Fragment extraction is achieved using Gray-level co-occurrence matrix. GLCM is the most utilized picture assessment structure due to its feature sets such as Contrast, Correlation, Energy, Homogeneity and Entropy [6].

\subsection{SVM Classifier}

Support-Vector Machines, also known as support-vector networks are discriminative classifier used in Machine Learning. SVM is an algorithm set for both regression and classification. The classification is done by the SVM with the hyper plane that splits the marked points into two categories. When new inputs are fetched in, they are mapped into model and are assigned to either of the 2 category based on which side they fall.

\subsubsection{Convolutional layers}

Convolutional layers are way different from the traditional neural networks as they preserve the spatial features rather than fully connected neural networks. The important features of this are they can preserve the structural features and are convolved again to retrieve without the loss of structural properties. The filters are 
basically small matrices of number that are multiplied by regions of input. Every pixel of input, the filter centre is imposed and multiplied to it, except for pixels having big neighborhood. The original depth of an input is increased by a filter [7].

\subsubsection{Pooling Layers}

It is added after convolutional layer. The two common pooling methods available are Average pooling and Max pooling [8]. It offers down sampling of feature maps separately as they are delicate to the position of features in the input so that a stride is setup.

\subsubsection{Fully connected neural network}

It forms the last few layers of the CNN. They connect every neuron in one layer with another and hence the name [9-11]. It performs classification based on the features extracted by the preceding layers. If the prediction matches the features high values are generated in the activation maps.

\section{Results and Discussions}

The proposed system was verified for both $\mathrm{CNN}$ and SVM classifier using 2D and 3D images. A sample 2D image is considered and it is analyzed using CNN and then preprocessed using Gaussian filter. After preprocessing, the image is segmented using Spearman algorithm then edge detection of segmented image using fuzzy clustering is performed as shown in Fig.2.

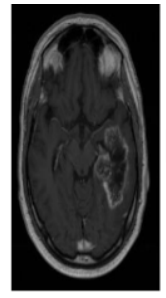

2D MRI Test image

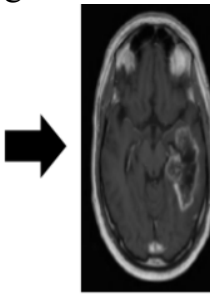

Pre-processed image

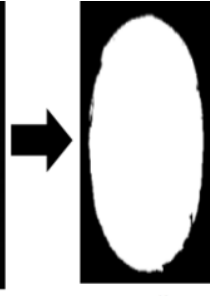

Segmented image

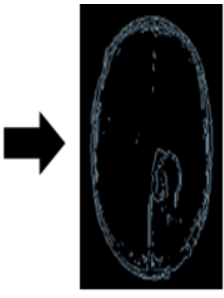

Edge detected image

Figure 2.Image processing of 2D test image

The sample image was analyzed using SVM classifier and then the results of CNN and SVM classifiers like accuracy and sensitivity are compared as shown in Fig.3.
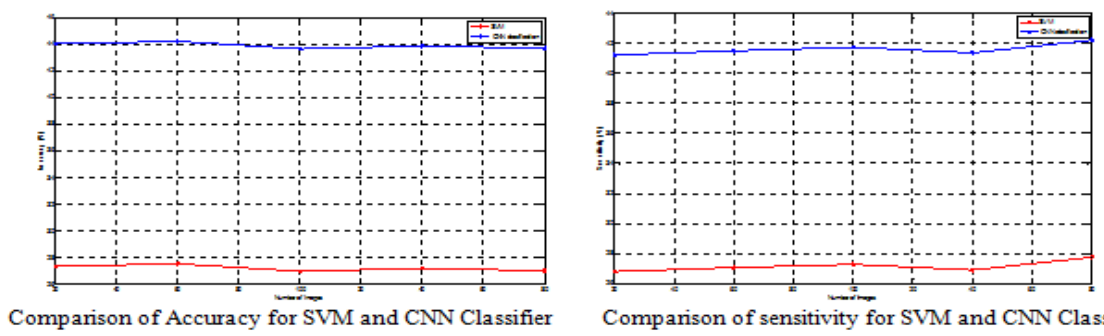

Comparison of sensitivity for SVM and CNN Classifier

Figure 3.Result analysis of test $2 \mathrm{D}$ image 
A sample 3D image is considered and it is analyzed using $\mathrm{CNN}$ and then preprocessed using Gaussian filter. The preprocessed image is segmented using Spearman algorithm. The DWT is used to obtain segmented image and then edge detection of the segmented image is performed as shown in Fig.4.

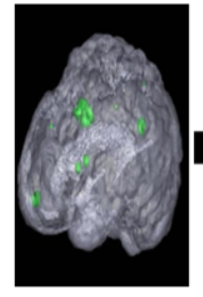

3D MRI Test image

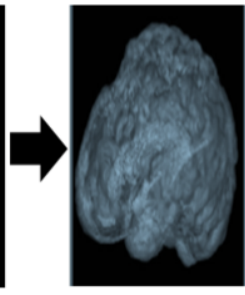

Pre-processed image

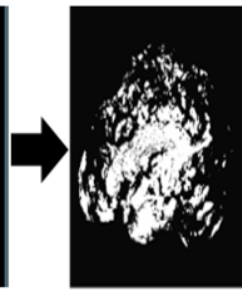

Segmented image

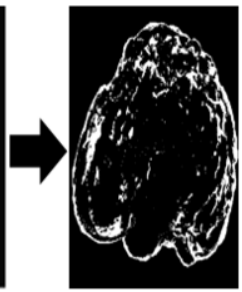

Edge detected image

Figure 4. Image processing of 3D test image

The sample image was analyzed using SVM classifier and then the results of CNN and SVM classifiers like accuracy and sensitivity are compared as shown in Fig.5
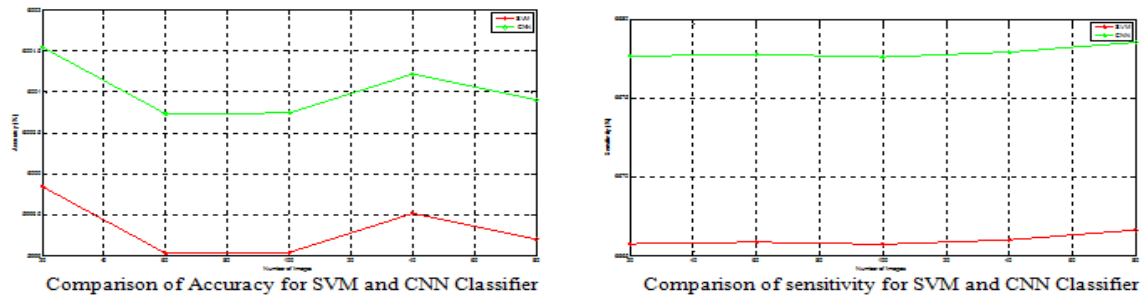

Figure 5.Result analysis of test 2D image

The result of 2D \& 3D images for CNN \& SVM classifier are compared in Table I.

Table 1. Comparison of $2 \mathrm{~d}$ and $3 \mathrm{~d}$ for svm and cnn classifier

\begin{tabular}{|l|c|c|c|c|}
\hline FEATURES & $\begin{array}{c}\text { 2D IMAGE } \\
\text { USING SVM } \\
\text { CLASSIFIER }\end{array}$ & $\begin{array}{c}\text { 2D IMAGE } \\
\text { USING CNN } \\
\text { CLASSIFIER }\end{array}$ & $\begin{array}{c}\text { 3D IMAGE } \\
\text { USING SVM } \\
\text { CLASSIFIER }\end{array}$ & $\begin{array}{c}\text { 3D IMAGE } \\
\text { USING CNN } \\
\text { CLASSIFIER }\end{array}$ \\
\hline Accuracy & 27 & 41 & 60 & 61 \\
\hline Sensitivity & 27 & 39 & 66.667 & 66.77 \\
\hline
\end{tabular}

On comparison, it was found that 3D image produces the better results than 2D. Similarly CNN produces accurate results than SVM for both 2D and 3D image inputs.

\section{Conclusion}

With the aid of Medical Imaging techniques automated using Machine Learning Algorithms, the existence of tumor cells during analysis is traced out.CNN classifier provides improved accuracy, sensitivity and specificity rate on comparison with the existing medical imaging systems in $3 \mathrm{D}$ which provides the exact size, depth and location of tumor with high accuracy. On a total of 9 2D MRI data, we trained the network with $62 \mathrm{D}$ MRI data and verified the results by giving $32 \mathrm{D}$ data as Test 
data.Here we used to train the neural network for producing the results of test image. Instead we can use the region growing algorithm to identify the tumor affected cell from the normal cells. Instead of using neural network we can use the latest Ant colony optimization and Genetic algorithms for producing the exact results.

\section{References}

[1] Alfonse M., and Salem, A B. M 2016, 'An automatic classification of brain tumors through MRI using support vector machine', Computer Science Journal, vol.40-issue-03..

[2] A.Rajendran, R. Dhanasekaran 2011, 'Fuzzy Clustering and Deformable Model for Tumor Segmentation on MRI Brain Image: A Combined Approach', International Conference on Communication Technology and System Design, pp 327-333.

[3] Bahadure N B, Ray A. K, and Thethi H. P. ,2017, 'Image analysis for MRI based brain tumor detection and feature extraction using SVM', International journal in Biomedical Imaging.

[4] Rachana Ramachandran.R.P, N.Mohanapriya, V.Banupriya, \&quot;A Spearman Algorithm Based Brain Tumor Detection Using CNN Classifier for MRI Images\& quot; ,International Journal of Engineering and Advanced Technology (IJEAT),Vol-8 Issue-6S, August 2019..

[5] Afsana Islam, MdFoisal Hossain, ChandanSaha 2017, 'A New Hybrid Approach for Brain Tumor Classification Using BWT-KSVM', International Conference on Advances in Electrical Engineering, vol.1, pp 5386-0869.

[6] B Muthuraj, V Mahesh, R Senthil 2016, 'A Hybrid Petri Net Approach for Polyethylene Terephthalate Bottle Manufacturing System',Journal of Computational and Theoretical Nanoscience.

[7] S. R. Balaji and S. Karthikeyan 2017 'A survey on moving object tracking using image processing', 11 th International Conference on Intelligent Systems and Control (ISCO), pp. 469-474.

[8] R.Senthil B.Muthuraj, .V.Mahesh (2015), 'Simulation Of Three Tank System Using Hybrid Perti Net For Batch Process' International Journal of Applied Engineering Research 4789-4798 Research India Publications.

[9] Abd El Kader Isselmou, Shuai Zhang, Guizhi Xu 2016, ‘A Novel Approach for Brain Tumor Detection Using MRI Images’, Journal of Biomedical Science and Engineering, vol.9, pp 44-52.

[10] V.D.Ambeth Kumar, Dr.M.Ramakrishnan, V.D.Ashok Kumar and Dr.S.Malathi (2015) "Performance Improvement using an Automation System for Recognition of Multiple Parametric Features based on Human Footprint" for the International Journal of kuwait journal of science \& engineering, Vol 42, No 1 (2015), pp:109-132.

[11] V.D.Ambeth Kumar, V.D.Ashok Kumar, Dr.S.Malathi and P.Jagaeedesh, (2014) "Intruder Identification using Footprint Recognition with PCA and SVM Classifiers" for the International Journal of Advanced Materials Research Vols.1345, PP 984-985 (2014) pp 1345-1349. [DOI:10.4028/www.scientific.net/AMR.984-985.1345] 